\title{
LOS RESTOS ANIMALES DEL YACIMIENTO DE TERRERA VENTURA
}

\section{(TABERNAS, ALMERIA)}

\author{
ANGELA VON DEN DRIESCH Y ARTURO MORALES
}

\section{INTRODUCCION SOBRE LA ALDEA ENEOLITICA DE TABERNAS}

E1 poblado de Terrera Ventura, situado en la iocali-. dad Almeriense de Tabernas, a unos $30 \mathrm{Kms}$. al norte de la ca pital, fue excavado durante tres campañas consecutivas (años de $1.972,1.973$ y 1.974 ) por el Arqué́logo Sr. D. Francisco Gusi Jener quien redactó los datos de esta introducción as 1 como la cronología proporcionándonos la oportunidad de estudiar los restos faunisticos hallados durante las excavacio-. nes. Por esta razón deseamos antes de nada agradecer al $\mathbf{S r}$. Jener la ayuda prestada en todo momento y que permitió una más correcta interpretacion de los resultados.

\section{CRONOLOGIA}

E1 yacimiento abarca un período cultural conocido de 700 años, aunque por el momento tanto el inicio del poblamien to del mismo, como su final, quedan muy imprecisos $y$ sin dataciones absolutas, por un lado a causa de la continuada superposición de estructuras arquitectónicas pertenecientes a los distintos momentos de habitat, que han barrido los res:tos más antiguos, y por otro lado debido a la intensa erosión que ha sufrido la superficie del yacimiento una vez fue aban donado y al misérrimo cultivo de secano que modernamente se ha practicado en el área del poblado, hoy dia ya en desuso.

Los recientes informes que hemos recibido de los aná1isis de C14 del Instituto de Quimica fisica "Rocasolano", Laboratorio de Geocronologia, constatan las siguientes fases cronologicas :

Fase I (?).- Horizonte quizás perteneciente al NeoIItico final. No posee fauna.

Fase II.- 2.700, Horizonte neo-eneolitico niforme.

Fase III.- 2.700-2.400, Horizonte eneolitico precampa 
Fase IV.- 2.400-2.000, Horizonte eneolitico, tipo Los Millares, con campaniforme e incipiente metalurgía del cobre en su momento final $(2.000-1.900)$.

\section{GENERALIDADES}

El número total de restos animales de Tabiernas supera excasamente los 4.000 (ver tabla 1). La gran mayoría la cons tituyen los vertebrados, especialmente los mamiferos. E1 peso total de éstos últimos asciende a $33,7 . \mathrm{Kg} .435$ fragmentos de huesos, es decir, el 12,7\% del número total de vertebra-dos no han podido ser identificados. Se trata en su mayoría de esquirlas óseas de animales domésticos principales.

A pesar de 10 reducido de la muestra, la variabilidad faunística es considerable. 24 especies de vertebrados y al menos 16 especies de moluscos (ver tabla 4) componen la lista. Los moluscos pueden considerarse como restos culinarios, a excepción de Glycimeris, que, dado el desgaste de sus valvas, pudieron haber sido recogidas en las playas.

La tabla 2 muestra el número de restos, el numero mínimo de individuos (NMI), y sus respectivos porcentajes por fases para cada especie de vèrtebrados. En la tabla 3 están representados los pesos $y$ algunos de sus porcentajes de 10 s diversos mamiferos por fases.

Antes de hacer ningún comentario sobre el significado de estos hallazgos, queremos señalar las similaridades generales que se observan entre las fases III y IV, tanto en el número de restos como en el NMI. Las diferencias que, en cam bio se ven con la fase II, pueden ser debidas únicamente al reducido tamaño de ésta (con menos del $8 \%$ del total de res-tos), $y$ por consiguiente no significativas para nuestro trabajo. Dado el reducido espacio de tiempo abarcado, resulta más lógico una relativa similaridad interfásica.

\section{REPRESENTACION Y DISTRIBUCION DE LOS DISTINTOS GRUPOS}

Examinando las tablas faunisticas observaremos, entre otros, los siguientes datos: 


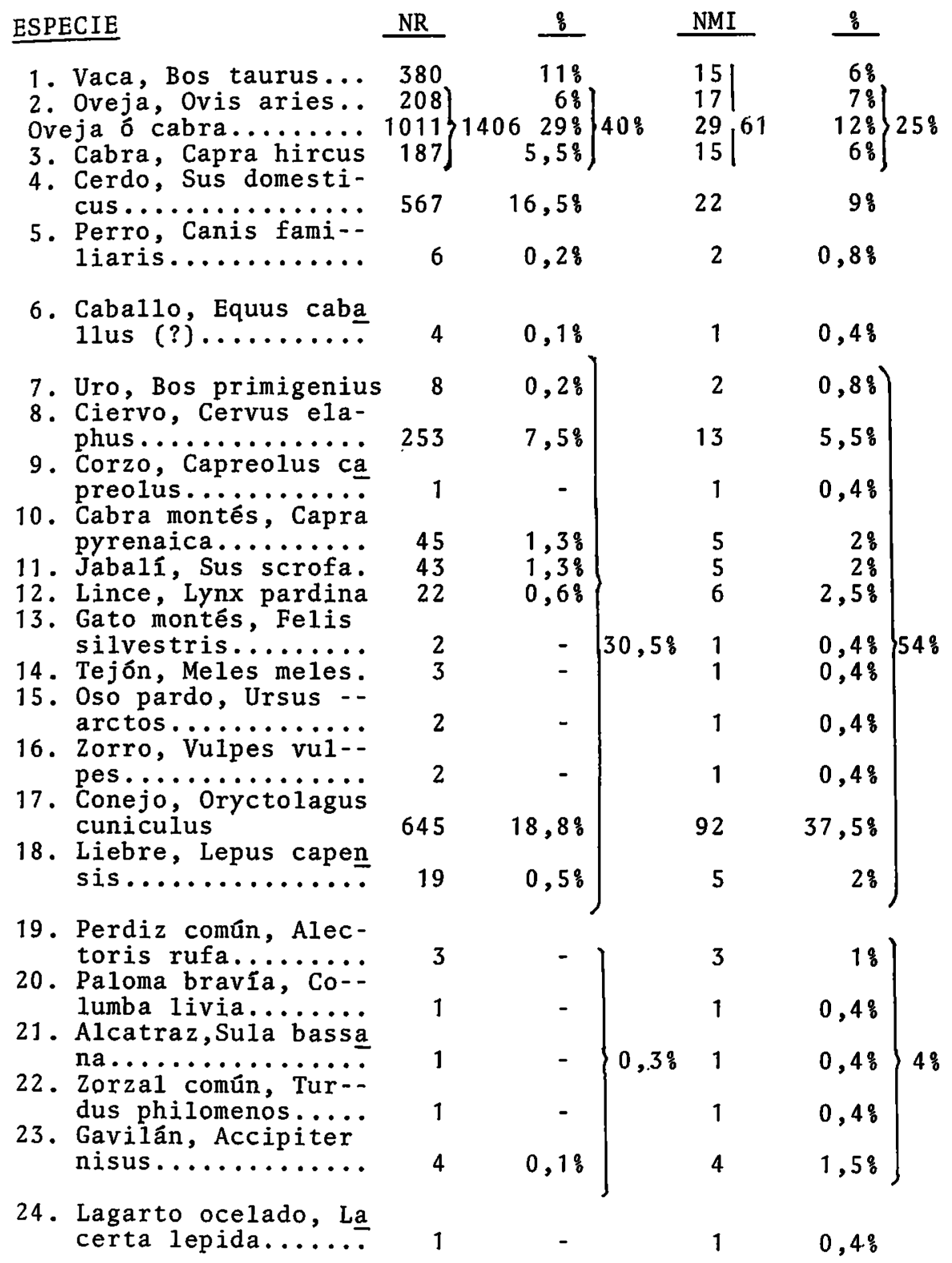


FASE II

ESPECIE

NR

8

NMI

$\underline{?}$

Vertebrados iden-

tificados......... 3419

$100 \%$

245

$100 \%$

Moluscos identifi-

cados (3)......... 149

Restos identifica

dos..................3568

Restos sin identi

ficar (4) ........ 435

Total restos ani-

males.......... 4003

Tabla 1 - Distribución en conjunto de los restos animales (vertebrados) por especies en e1 yacimiento de Tabernas.

(1) $\mathrm{NR}=$ Número de restos

(2) NMI = Número mínimo de individuos

(3) Ver tabla 4 .

(4) Sólo vertebrados.

1) Las especies domésticas son más abundantes que las salvajes, como suele ocurrir en todos los yacimientos humanos de finales del Neolitico en la Península Ibérica. Las especies silvestres, no obstante, se hallan ampliamente representa-das. Según los porcentajes del NMI este grupo representa el 588 del total, es decir, el doble aproximadamente de su valor si consideramos el número de restos sólo. Por otra parte, el valor real de los animales económicamente importan-tes del yacimiento - Vaca, oveja, cabra, y cerdo, pero

FASE I I

ESPECIE

Vaca, Bos taurus... 20

Oveja, Ovis aries... 13

Oveja 6 cabra......

Cabra, Capra hircus

Ciervo, Cervus ela-

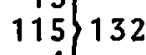

phus

Lince, Lynx pardina 1

Conejo,Oryctolagus

cuniculus......... 37

Liebre, Lepus ca-

pensis............

Gavilán, Accipiter

nisus........... 1

Tota1 identificados 222

Sin identificar.... 62

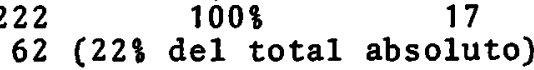

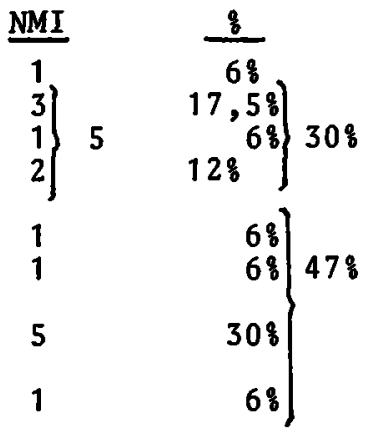

Total absoluto..... 285

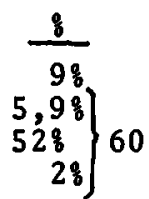

$4 \%$
$0,3 q$

$\left.\begin{array}{l}0,38 \\ 178 \\ 0,38\end{array}\right\} 21,58$

0,38

1

$6 \%$
$00 \%$ 


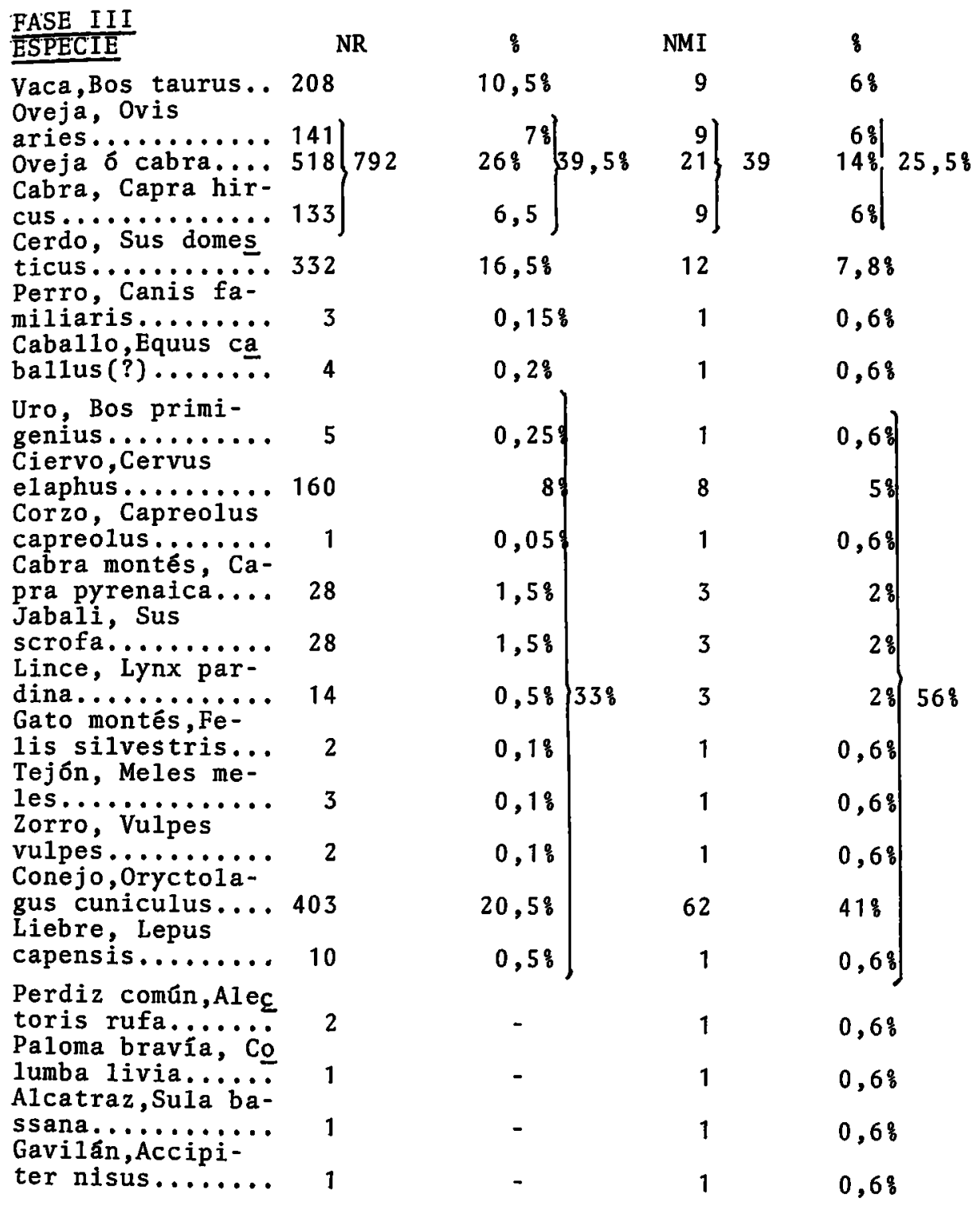


FASE I I

ESPECIE

$\underline{N R}$

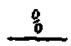

$\underline{N M I}$

$\stackrel{?}{?}$

Total identifi-

cados.......... 2000

Sin identificar. 101

$(4,8 \%$ del total absoluto $)$

$100 \%$

Tota1 absoluto.. 2101

Tabla 2. Distribución de vertebrados domésticos y silvestres por fases.

FASE IV

ESPECIES

Vaca,Bos taurus. 152

Oveja, Ovis

aries......... 54

Oveja ó cabra... 378$\} 482$

Cabra, Capra hi $\underline{r}$

cus.......... 50

Cerdo, Sus do-

mesticus....... 214

Perro,Canis fa-

miliaris....... 3

Uro, Bos primi-

genius........ 3

Ciervo, Cervus

elaphus....... 84

Cabra montés, $\mathrm{Ca}$ -

pra pyrenaica... 17

Jabali, Sus

scrofa........ 15

Lince, Lynx par-

dina.......... 7

Oso pardo, Ursus

arctos.......... 2

Conejo,Oryctola-

gus cuniculus

Liebre, Lepus ca-

pensis........ 8

Perdiz común,

Alectoris rufa.. 1

Zorzal común, Tur

dus philomenos.: 1

Gavilán,Accipi-

ter nisus...... 2

Lagarto ocelado, Lacerta lepida

NR

$\frac{?}{12,5 \%}$

NMI

$$
4,5 \%
$$

$31,5 \% 40 \%$

$4 \%$

$18 \%$

$0,25 \%$

$0,25 \%$

\begin{tabular}{r|r}
$7 \%$ & 4 \\
$1,5 \%$ & 4
\end{tabular}

1,5

$1,25 \% 28,5 \% \quad 2$

$0,5 \%$

$0,1 \%$

$17 \%$

$0,5 \%$

5

$\frac{8}{6,5 \%}$

5

$7\} 17$

5

$6,5 \%$

$10,5 \%$

$1,3 \%$

$1,3 \%$

$5 \%$

$2,5 \%$

$2,5 \% 52,6 \%$

$2,5 \%$

$1,3 \%$

$33 \%$

4\%

$\left.\begin{array}{l}1,3 \% \\ 1,3 \% \\ 2,5 \%\end{array}\right\} 5,3 \%$

$0,1 \div 0,3$

2

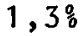


FASE I I

ESPECIE

$\underline{\text { NR }}$

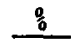

NMI

$\stackrel{q}{8}$

Total identifica

dos................ 1197

Sin identificar.. 271

$100 \% \quad 76$

$100 \%$

Total absoluto... 1468

Tabla 2 (continuación). Distribución de vertebrados domésticos y silvestres por fases.

tmabiên el ciervo - retrocede al considerar los purcentajes re`ativos del NMI. Sabemos, sin embargo, que no debemos tomar estos indices como base para la representación específica de animales determinados en yacimientos, ya que producen a menudo resultados contradictorios.

E1. valor de la aproximación de1 NMI a1 número real de individuos es un factor que disminuye en proporción directa con el aumento del número de restos (NR). En otras palabras, las especies más importantes económicamente se hallan casi siempre infrarepresentadas porque aumenta, en medida similar a su número, el número de piezas recuperadas de sus esqueletos en el yacimiento. Este fenómeno causa la disminución aparente que observamos al considerar los porcentajes del NMI y distorsiona la representación real de todas las especies. De ésta manera vemos que en nuestro yacimiento todas las formas representadas por un reducido número de hue sos aumentan su valor si en lugar de considerar los porcentajes relativos del NR tomamos aquelios del NMI, Tambien ocurre hasta cierto punto un hecho parecido con el conejo, pues si bien es abundante siempre y según el número de restos aumenta su valor real al considerar los porcentajes del NMI ya que casi siempre recuperamos de ésta especie las mismas piezas características (pelvis, tibias, ulnas, etc...), factor éste que causa una disminución del NR/individuo presente. (Vemos por ejemplo que si el porcentaje del conejo según el $N R$ es de $18,8 \%$, según el NMI aumenta hasta el -.$37,5 \%$. Es muy importante tener en cuenta todos estos datos a la hora de la evaluación para corregir las discrepancias producidas por estos métodos de aproximación a la biomasa total representada. 


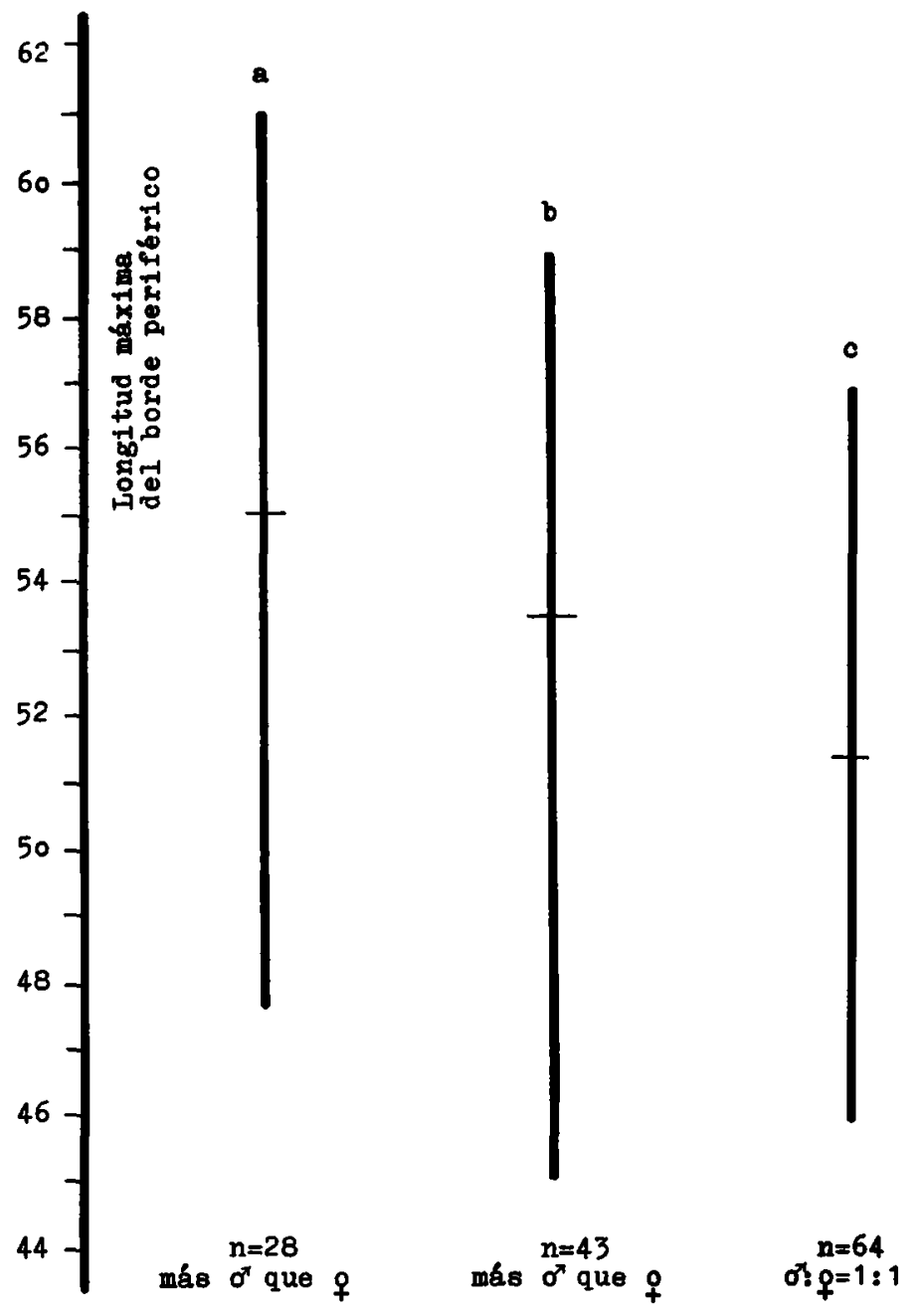

Diagrama: 1 Representación gráfica del tamaño comparatiro de 3 poblaciones prehistóricas de ciervos de la Peninsula ibérica oegún la longitud de la primera falange.

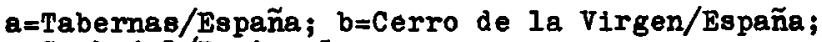
$c=$ Zambujal/Portugal 


\begin{tabular}{|c|c|c|c|c|c|c|c|}
\hline ESPECIE & FASE I I & FASE II I & $\because$ & FASE IV & 8 & TOTAL & 8 \\
\hline \multicolumn{8}{|l|}{ Vaca,Bos. } \\
\hline taurus...... & 525 & 5805 & 28,28 & 3125 & 26,88 & 9455 & $28 \%$ \\
\hline aries....... & 110 & 1090 & & 565 & & 1765 & \\
\hline Crabra, Capra. & 400 & 3510 & $28,5 \%$ & 2495 & $30 \%$ & 6405 & $29 \%$ \\
\hline $\begin{array}{l}\text { hircus ...... } \\
\text { Cerdo, Sus do }\end{array}$ & 35 & 1275 & & 460 & & 1770 & \\
\hline mesticus ... & 115 & 2940 & 14,38 & 1950 & $16,7 \%$ & 5005 & $15 \%$ \\
\hline familiaris.. & - & 30 & - & 20 & - & 50 & - \\
\hline Caba110,Equus & - & 200 & $1 \%$ & - & - & 200 & - \\
\hline $\begin{array}{l}\text { Uro,Bos primi } \\
\text { genius........ } \\
\text { Ciervo,Cervus }\end{array}$ & - & 288 & $1,4 \%$ & 155 & $1,3 \%$ & 443 & $1,3 \%$ \\
\hline $\begin{array}{l}\text { elaphus (1)... } \\
\text { Corzo, Capreo }\end{array}$ & 190 & 3720 & $18 \%$ & 1770 & $15,2 \%$ & 5680 & $17 \%$ \\
\hline $\begin{array}{l}\text { lus capreolus } \\
\text { Cabra montés, } \\
\text { Capra pyrenai }\end{array}$ & - & 15 & - & - & - & 15 & - \\
\hline Jabaì, Sus sí & 0 & 555 & 2,78 & 420 & $3,6 \%$ & 975 & $2,9 ?$ \\
\hline fa........... & - & 400 & 28 & 325 & $2,8 \%$ & 725 & $2,2 \%$ \\
\hline \multicolumn{7}{|l|}{$\begin{array}{l}\text { Gato montés, } \\
\text { Felis silvest }\end{array}$} & $0,6 \%$ \\
\hline \multicolumn{8}{|l|}{ Triśón, Meìis } \\
\hline \multicolumn{8}{|l|}{ Oso pardo,Ursus } \\
\hline \multicolumn{8}{|l|}{$\begin{array}{l}\text { arctos......... } \\
\text { Zorro,Vulpes }\end{array}$} \\
\hline \multicolumn{8}{|l|}{ Vulpes } \\
\hline $\begin{array}{l}\text { lagus cuniculus } \\
\text { Liebre, Lepus }\end{array}$ & 40 & .535 & 2,68 & 225 & $1,9 \%$ & 800 & 2,3 \\
\hline capensis...... & 12 & 53 & - & 35 & - & 100 & - \\
\hline TOTAL........ & 1437 & 20609 & $100 \%$ & 11650 & $100 \%$ & 33696 & \\
\hline $\begin{array}{l}\text { Tabla 3. Pesos } \\
\text { (1) Los pesos d } \\
\text { grs. Fase I }\end{array}$ & $\begin{array}{l}\text { de } 1 \text { as } \\
\text { IV } 130\end{array}$ & $\begin{array}{l}\text { astas so } \\
\text { grs. ye }\end{array}$ & $\begin{array}{l}\text { Fase } \\
\text { total }\end{array}$ & $\begin{array}{l}\text { I I } 80 \mathrm{grs} \\
\text { es de } 69\end{array}$ & $\begin{array}{ll}95 & \text { grs }\end{array}$ & III & \\
\hline
\end{tabular}


2) Los ovicaprinos demésticos constituyen, por pesos, casi e1 $30 \%$ de 1 a muestra. Les siguen 1a vaca con un $28 \%^{\circ} y$ en tercera posición el ciervo con el $17 \%$ del peso total. E1 cerdo representa el $15 \%$ del total (ver tabla'3.)

\section{ESPECIE FASE II FASE III FASE IV TOTAL}

1. Pelecípodos

Arca noe.............

Cerastroderma ( $=$ Car

dium) sp.......... -

Glycimeris sp...... 1

Ostrea edulis...... -

Pecten jacobaeus... -

Spondylus sp........

$\begin{array}{rrrr}- & 2 & - & 2 \\ - & - & 1 & 1 \\ - & 36 & 27 & 64 \\ - & 1 & - & 1 \\ - & 1 & - & 1 \\ 1 & 3 & - & 3 \\ - & - & 1 \\ - & - & 1 & 1 \\ 1 & 4 & - & 4 \\ - & - & - & 3 \\ - & 4 & - & 4 \\ - & 35 & 13 & 48 \\ - & 4 & 2 & 6 \\ & 5 & 3 & 8 \\ - & 3(1) & - & 3 \\ 3 & 100 & 49 & 15.2\end{array}$

2. Gasteröpodos

Cypraea sp (?) ...

Monodonta turbinata

Murex brandaris....

Nassa sp............

Purpura haemostoma.

Patella sp...........

Triton nodifer.....

Pu1monados..........

3. Cefalópodos

Loligo sp...........

100

49

15.2

Tabla 4 - Distribución general de los moluscos por niveles. (1) Los tres fragmentos pertenecen a un mismo individus.

Sospechamos al igual que en el anterior apartedo, que estos pesos no deben ser buenas aproximaciones de 105 verdaderos valores, y en especial creemos que tanto la vaca como el ciervo deben hallarse infrarepresentados.

De cualquier modo, los pesos siguen siendo el mejor método para representar graficamente el auténtico papel de cada una de las diferentes especies en el aporte cárnico a la dieta de un determinado yacimiento.

3) La proporción de la cabra y la oveja, que, salvo en e1 es trato II, se mantiene siempre en 1::1 aproximadamente. Este dato, normal a primera vista nos va a resultar de gran valor cuando intentemos dar una pequeña vision ecologica de lo que fué el yacimiento de Tabernas en 1a Época en que se 
Jesarrolló la cultura aquí estudiada.

4) La escasa representación del cabal10. Debido al pequeño material disponible poco podemos decir salvo que se trata de un auténtico caballo y no un asno, tras el examen de un molar superior.

5) Los demás vertebrados no mamíferos representan una fracción insjgnificante dol material (ver tabla 1) tanto en NR como en NMI.

\section{DATOS BIOLOGICOS COMPLEMENTARIOS}

Basándonos en los caracteres moriológicos de los huesos homos reconstruido algunos datos complementarios sobre las distintas especies que a continuación detallaremos. Se refieren especialmente a tres capitulos principales: grupos cronológicos, dimorfismo sexual y alzada.

En las tablas 5 y 6 se hallan representados por -grupos de edad de los ovicaprinos $y$ del cerdo según las fechas de aparición, reemplazo y desgaste de las piezas dentatias mandibulares. Como puede verse, todos los cerdos estudia dos son menores de 2 años, y por 10 tanto, no existe ningún individuo adulto en la muestra de mandibulas. El único adulto que hemos encontrado en todo el yacimiento ha sido descubierto al encontrar una porción proximal de un fémur que tenía el caput femoris $y$ el trocanter major fusionados ya a -. la diáfisis.

En la oveja y la cabra el cuadro es distinto. Dominan los adultos (más de 18 meses de edad) pero los individuos menores de $11 / 2$ años representan la tercera parte del total. En la fase III, además, existen mayor número de individuos jóvenes que en la fase IV.

\begin{tabular}{lcccc} 
GRUPo DE EDAD & $\begin{array}{c}N^{\circ} \text { de mandi- } \\
\text { bulas } \\
\text { (III) }\end{array}$ & $\begin{array}{c}\text { NMI } \\
\text { (III) }\end{array}$ & $\begin{array}{c}\text { Node mandi }^{\circ} \text { bulas } \\
\text { (IV) }\end{array}$ & $\begin{array}{r}\text { NMI } \\
\text { (IV) }\end{array}$ \\
\hline $\begin{array}{l}\text { Menores de 2 años. } \\
\text { Mayores de 2 años. }\end{array}$ & 22 & 16 & 7 & 5 \\
\hline
\end{tabular}




\begin{tabular}{|c|c|c|c|c|c|c|c|c|}
\hline & FASE & I I & FASE & III & FASE & IV & TOTAL & EDAD \\
\hline MAND I BULA & $I(1)$ & $\mathrm{D}(2)$ & $\underline{I}$ & _D & $\underline{I}$ & $\underline{D}$ & & \\
\hline $\begin{array}{l}\text { M1 erupcionando.. } \\
\text { M1 presente; M2 }\end{array}$ & - & - & 1 & - & - & - & 1 & \\
\hline 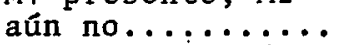 & - & 1 & 5 & 1 & - & - & 7 & | \\
\hline $\begin{array}{l}\text { M2 erupcionando.. } \\
\text { M2 presente; M3 }\end{array}$ & - & - & 3 & 2 & 1 & 3 & 9 & 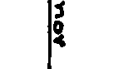 \\
\hline aún no........... & 1 & 1 & 3 & 5 & 1 & 1 & 12 & \\
\hline M3 erupcionando.. & 1 & - & - & 2 & 1 & - & 4 & $\begin{array}{l}\text { aprox } \\
18 \text { mese }\end{array}$ \\
\hline M3 $(+) \ldots \ldots \ldots \ldots$ & 1 & - & 9 & 11 & 7 & 5 & 33 & \\
\hline M3 $(++) \ldots \ldots \ldots$ & - & - & 4 & 5 & 3 & 2 & 14 & $\xi$ \\
\hline M3 $(+++) \ldots \ldots \ldots$ & - & - & 5 & 2 & 1 & 2 & 10 & $\int_{0}^{R}$ \\
\hline TOTAL........... & 3 & 2 & 30 & 28 & 14 & 13 & 90 & \\
\hline
\end{tabular}

Tabla 5. Grupos de edad en la oveja y la cabra, basados en el estudio de las mandibulas.

Es decir, una proporción de jóvenes/adultos de -aproximadamente $4: 5-6$ en la fase IIJ y de $1: 2$ en Ia fase IV.

E1 ciervo de la fase II, es un animal adulto, como los 4 individuos de la fase IV. En la fase IIl, tenemos dos individuos infantiles, dos juveniles y cuatro adultos. La vaca de la fase II es adulta. En la fase III, de 9 individuos, tres al menos son menores de 2 años $y$ en la fase IV, hay también al menos un individuo juvenil (M1 erupcionando).

El dimorfismo sexual es evidente cn todos los herbívoros, menos en el jabalí. Aunque también es patente en el cerdo, nuestra escasez de adultos no nos ha permitido corroborarla. La proporción de los sexos, basada en las -primeras falanges es de 2 hembras: 1 macho para 1 as ovejas y cabras. Igualmente, de acuerdo con éstas piezas, la propor ción en el ciervo de la fase III es de 3 hembras: 4 machos $\vec{y}$ de 1 hembra: 4 machos en la fase IV. En la fase II no sabemos si el único individuo contabilizado es macho o hembra aunque aparecieron en esta fase fragmentos de astas. 


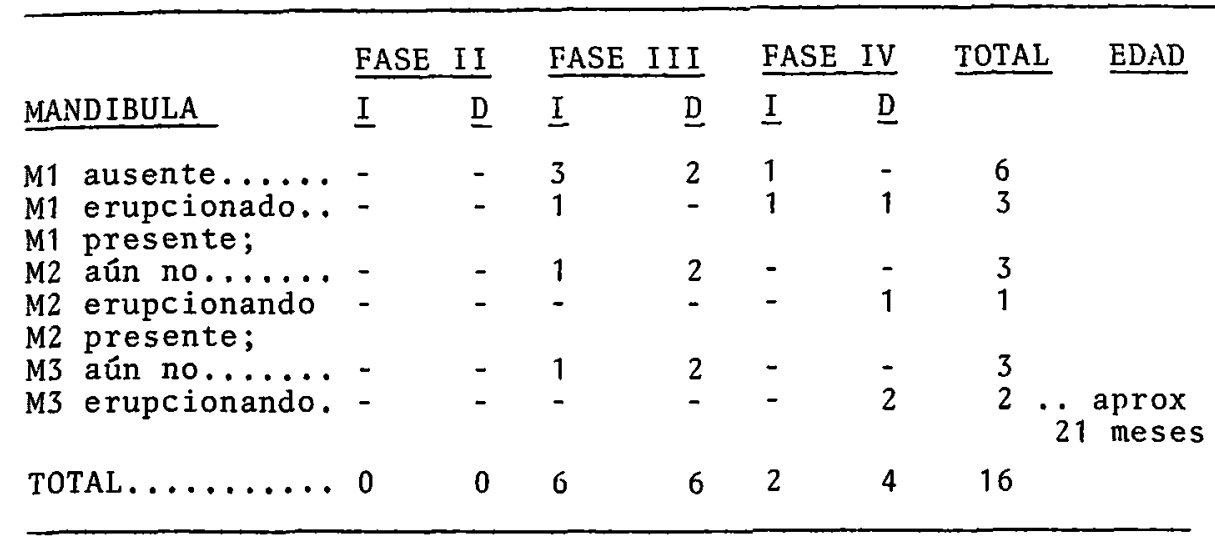

Tabla 6. Grupos de edad en el cerdo, basados en el estudio de las mandíbulas.

En la vaca de la fase III, y según los metapodios, tenemos una proporción de 2 hembras: 1 macho y según las pel vis (acetábulos), una proporción de 1 hembra : 1 macho. En la fase IV sólo podemos aventurar que hay al menos un macho y una hembra. La mayoría de los huesos recuperados de cabra montés pertenecen a machos. Debido a que algunas de las hembras e individuos juveniles de ésta especie pueden haberse incluído con los óvicaprinos domésticos, resulta más conveniente no aventurar las proporciones de los sexos ni tampoco la estructura cronológica de la muestra en esta especie.

En 10 que a tamaño se refiere hemos podido constatar algunos hechos despues de haber medido los distintos elementos óseos de cada especie:

1 - Las vacas de Tabernas son animales de talla extremadamente reducida, no sólo en relación con sus parientes centroeuropeos prehistóricos, sino con sus propios contemporáneos de yacimientos ibéricos como por ejemplo Cerro de la Virgen y Zambujal.

2- Por el contrario, los ciervos son, a pesar de la relativa abundancia de machos en las muestras, individuos de gran tamaño que superan ampliamente a las subespecies hispáni cas actuales. El tamaño del ciervo de Tabernas en comparación con otro yacimiento andaluz y uno portugués de la misma epoca se observa en el diagrama 1 . 
3- Las ovejas y las cabras domésticas de Tabernas son, como en la mayoría de los yacimientos penínsulares, formas esbeltas de talla pequeña comparadas con las ovejas $y$ cabras prehistoricas centroeuropeas. Los cuernos de las cabras eran comprimidos y delgados, en forma de cimitarra, de igual morfología que las cabras actuales españolas. Algunas ovejas hembras eran ya mochas.

4- Aunque la mayoría de los restos de cerdo son de animales jovenes, 10 que dificulta la reconstrucción de su tamaño, sabemos de cualquier modo que los cerdos eran peque ños $y$ esbeltos, parecidos a las razas semisilvestres actuales de algunos puntos del Sur de la Peninsula.

5- Del caballo podemos decir que se trata de un -típico ejemplar hispánico por cuanto respecta a su falange: gracil y de talla mediana. Posee una primera falange con una anchura proximal sensiblemente mayor que la anchura distal. De tratarse de un animal domestico sería la primera cita española de esta especie. a machos.

6- Los restos de uro reconocidos pertenecian todos

7 - Los huesos de perro pertnecen a dos individuos adultos de pequeña talla que se pueden comparar con el tipo del "perro de los palafitos" (C.f. forma palustris) -. suizo.

8--En conjunto, todos los mamiferos salvajes son de proporciones grandes. Las cabras monteses y los jabalíes superan en tamaño a las formas actuales. Los linces $y$ gatos monteses tienen valores equivalentes a los de yacimientos de la misma época. Estos últimos podrían pertene-cer a la subespecie meridional tartessia.

9 - En cuanto a los conejos podemos decir que son ligeramente menores que los individuos de Cerro de la Virgen, aproximándose pues a las formas extantes.

Entre los huesos de Tabernas tenemos algunas piezas con muestras de patología osteomorfologica. Asi por ejemplo, la figura 6 muestra un metatarso de oveja que, tras sufrir una ficactura del cớlilo medial, se soldó posteriormente a distinto nivel. Una mandíbula de oveja $\delta$ cabra de la fase IV muestra una periostitis alveolar avanzada; también aparecieron dos vértebras costales de éstas especies con una defirmación lateral del cuerpo vertebral. Tres primeras falanges de ciervo (dos de ellas en las figuras $2 a$ y $2 b$ ) poseen exostosis en sus bordes periféricos, una de ellas en 
estado muy avanzado.

Algunas primeras falanges de ciervo, oveja y cabra se hallan rebajadas $y$ pulidas artificialuente (ver riguras 3 y 4). Parece que son los primeros estados en la manufactura de ídolos megaliticos que estudiaron anteriormente Topp \& Arribas (ver Topp \& Arribas, 1965). Algunas de éstas primeras falanges tráajadas de pequeños ungulados (cerdo, oveja, cabra), procedentes de las primeras excavaciones en Terrera Ventura se hallan representadas en la păgina 72 de su artículo. Estas falanges se distinguen de las nuestras, en que todas ellas muestran profundas incisiones en los -extremos distales. En la figura 9 de su trabajo aparece la falange de un équido, que no es de un caballo, con una incisión triangular en el borde proximal $y$ a 1 a cual no parece que se aluda en el texto. Para más detalles sobre el significado zoológico de esta pieza, ver von den Driesch (1972 pág. 117).

Estos mismos autores describen además punzones hechos de hueso $y$ que, en opinión de los investigadores "... are mostly made out of ulnas and of metapodials of sheep or goat" (pág. 76). Los dibujos de la figura 5, sin embargo, no coiniden plenamente con estas conclusiones ya que el punzón $n^{\circ} 62$, por ejemplo, es claramente una tibia de oveja ó cabra $y$ el $n^{\circ} 64$ es una ulna de lince hispánico (Lynx pardina). Los restantes punzones (NS 60-66, según los autores) son difíciles de determinar examinando tan sólo esta lámina que nos muestran.

Desconocemos en este momento si en las nuevas excavaciones de donde procede el material que aquí estudiamos se han encontrado nuevas falanges ó restos óseos animales convertidos en implementos humanos del tipo que Topp \& Arribas han descrito.

\section{FAUNA Y VEGETACION}

Las faunas o asociaciones de macromamiferos halla-dos en yacimientos arqueológicos no suelen resultar buenas bases para la reconstrucción de ambientes pretéritos donde se desarrollaron determinadas culturas. En particular, suelen contener pocas de las 11 amadas "especies-indices" con-finadas a un sólo biotopo. De todas formas, la variedad del conjunto faunistico de Tabernas nos ha permitido hasta un cierto punto imaginar un habitat diferente a la actual estepa árida que hoy ocupa esta zona, donde se desarrollo la cultura neolítica de este poblado. Sabemos, por ejemplo, -- 
que las grandes formas como el uro, el ciervo ó el jabalí, necesitan bosques ó tierras ricas donde pueda prosperar la vegetación para poder establecerse. Sospechamos que por los valles, hoy secos, pudieron transcurrir cursos de agua que permitieron la formación de arboledas a sus orillas. Probablemente las laderas de las montañas, que alcanzan unas alturas máximas de $800 \mathrm{~m}$., poseían igualmente árboles. No sabemos con certeza que especies vegetales existieron, aunque los caducifolios, en general se hallarían confinados a los valles y márgenes de ríos. El resto estaria constituído por un bosque mixto, abierto en algunas zonas, con especies más mediterráneas y más xéricas. No podemos creer, que, como indica Lautensach (1969, pág. 616), esta franja de tierra constituyese en el pasado una estepa natural, aunque sería muy interesante realizar un análisis polínico del yacimiento. Aventurarnos a citar especies que pudieron haber existido en Tabernas resultaria poco menos que imposible con el material de que disponemos.

La presencia de la cabra montés, en condiciones -normales, indica una cobertura vegetal más o menos densa -. (árboles, arbustos o bosque mixto, abierto) en las laderas de las montañas. Las hembras e individuos jóvenes suelen = encontrarse a menudo en las cotas más altas de la masa fo-restal, donde no son raras en ocasiones las pedrizas. A menudo, en estos mismos biotopos entran especies muy diver-sas, como pueden ser el conejo, la paloma bravía y la perdiz común. Normalmente, estos tres animales denotan condiciones mucho más mediterráneas que las cabras, hallándose, en general en regiones de menos árbol y más arbusto (caméfitos aromáticos), donde no aparece nunca la cabra montés.

Tabernas es actualmente demasiado pobre para mantener la presencia de ciervo, cabra montés, jabalí y uro. No estamos seguros si el corzo puede asimilarse a éste ensamblaje faunístico, ya que el único resto encontrado en nuestro yacimiento, (un fragmento de asta calcinada) pudo -haber sido transportado desde otro lugar. Caso de no ser -. así, el corzo reforzaría la hipotesis de un bosque caducifolio o al menos húmedo ya que es mucho más selectivo en este respecto que el resto de las especies aquí descritas.

Una prueba indirecta de la riqueza faunistica de -Tabernas, y por 10 tanto de su riqueza vegetal, la constituyen la variedad de los carnivoros aparecidos. Lince, gato montés, tejón, oso y zorro, se hallan más ligados a la presencia de presas adecuadas que a una determinada cobertura vegetal. La presencia de todos éstos animales, y el tamaño 


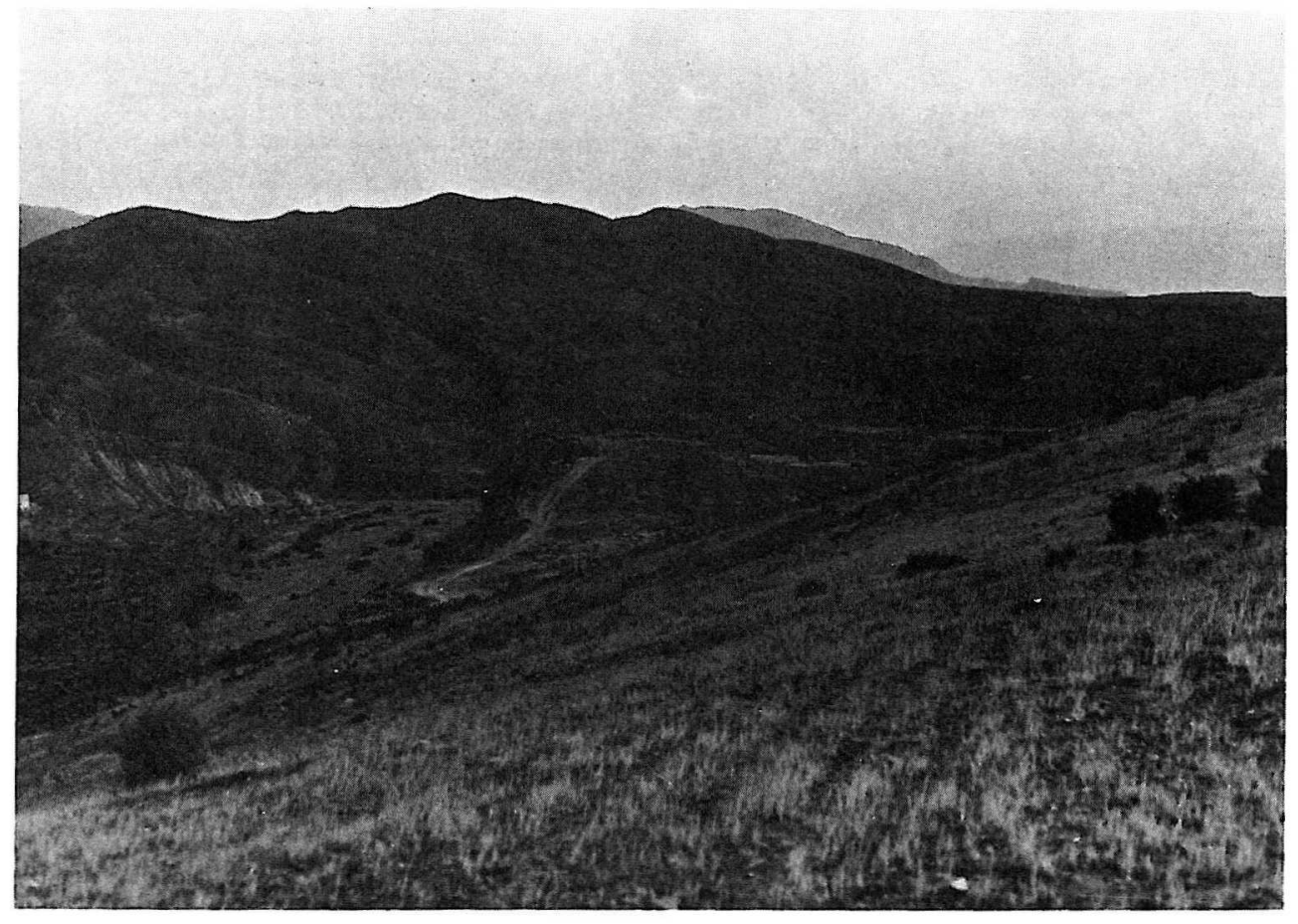



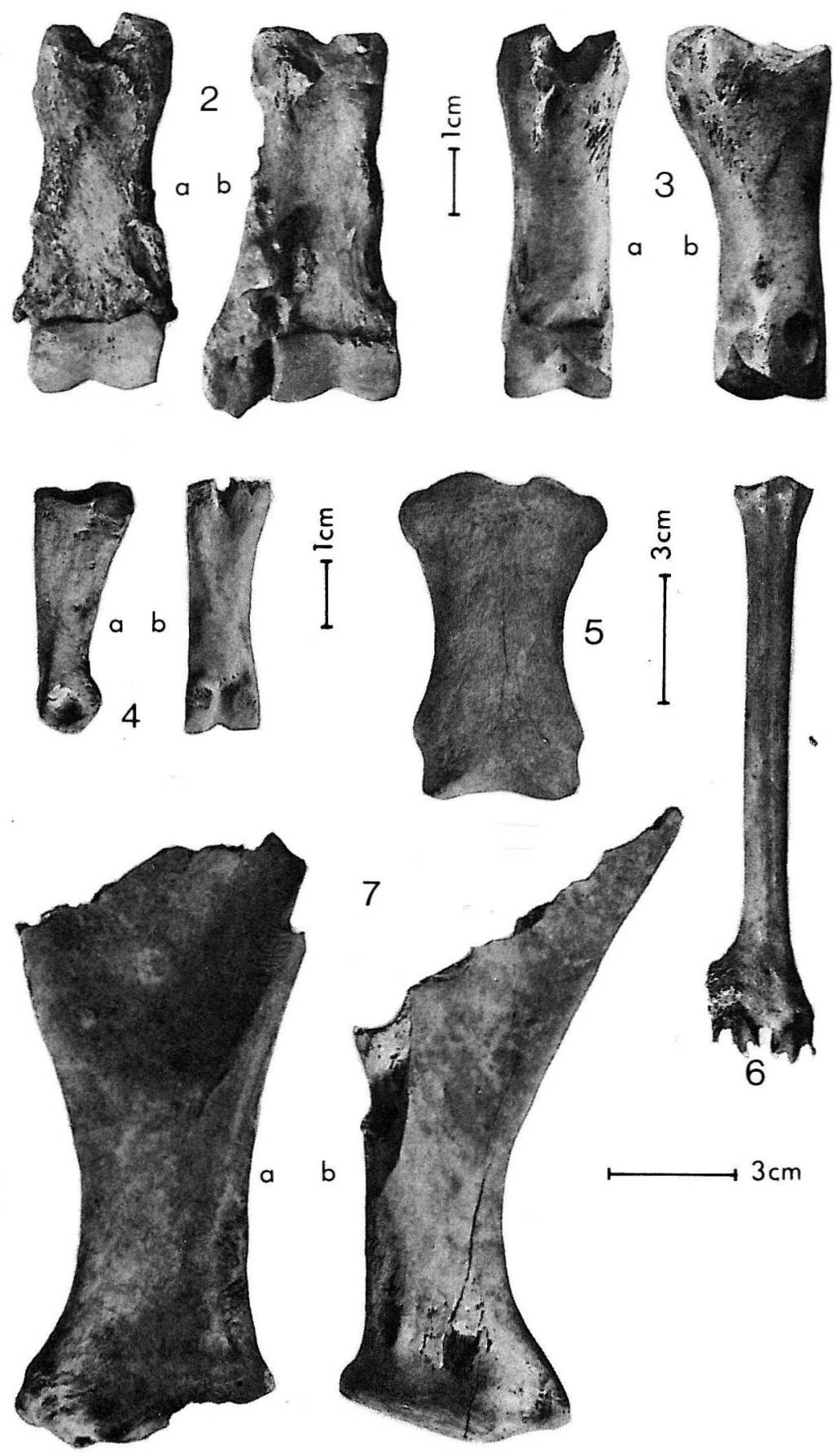
de algunos de ellos nos indican que los fitofagos debieron ser abundantes en este tiempo.

El perro, como animal doméstico, nada aporta al con junto faunístico, pues se halla ligado al hombre y muchas veces forzado a subsistir en condiciones precarias, que en nada tienen porqué asemejarse a la de sus agriotipos.

En contraste con los animales silvestres, el tamaño de las especies domésticas es siempre reducido y refleja en especial el sometimiento de éste grupo en conjunto a un régimen de vida diferente que no es otro que el impuesto artificialmente por el hombre.

Con toda certeza, la destrucción de este rico habitat a manos del hombre fué un proceso rápido. Tanto por medio de su acción directa, talando y deforestando zonas para el establecimiento de campos de cultivo, como con la - introducción de sus especies domésticas, el hombre creó la estepa árida que hoy conocemos. La degradación de la cobertura vegetal dejó tras de sí suelos frágiles a la erosión, que dado la lentitud de los procesos en las zonas cálidas, no pudieron recuperarse. A éste respecto debemos recordar la acción particularmente nociva de la cabra, tan abundante en Tabernas como la oveja, $y$ que fué sin duda factor importante en este proceso.

No sabemos a ciencia cierta si en el curso de estos últimos 5000 años ha habido cambios importantes en el régimen pluviometrico de esta región.

La zona, costa desde Alicante hasta Adra, constituye hoy en día el área más seca de toda la Península ibérica, con unos indices anuales que, salvo raras ocasiones, no superan nunca los $300 \mathrm{~mm}$. El"bache" estival es especialmente agudo. Pero aún cuando estos indices hubiesen permanecido constantes, la propia vegetación habría impedido una pérdida incon trolada de agua, contribuyendo así al mantenimiento de un microclima más húmedo, tanto por medio de la evapotranspiración, como por el mantenimiento permanente a un nivel accesible a los demas organismos de la capa freática, factores que hubiesen sido suficientes para el establecimiento de una fauna como la que aquí hemos brevemente descrito. 


\section{BIBLIOGRAFIA}

DRIESCH, A. von den \& J..Boessneck- Vorgenschichtliche Kanin chen aus zwei südspanischen Siedlungshügeln - Säugetierkd1. Mitt. 18: 127, 151, 1970 .

DRIESCH, A. von den - Osteoarchäologische Untersuchungen auf der Iberischen Halbinsel - Studien über frühe Tierk nochenfunde von der Iberischen Halbinsel 3. Müchen,, 1972 .

DRIESCH, A. con den \& J. Boessneck-Fie Tierknochenfunde vom Castro do Zambujal - Studien über frühe Tierknochen funde von der Iberischen Halbinsel 5, Müchen (en prensa).

LAUTENSACH, H. Iberische Halbinse1 - München, 1969 .

TOPP, C. \& A. Arribas- A Survey of the Tabernas material lodged in the Museum of Almeria - Bull, Inst. Archaeol., 5 : $69-89$, London.

\section{TABLA DE FIGURAS}

Fig. 1. Vista general del yacimiento de Terrera Ventura. Foto F. Gusi Jener.

Fig. 2. Falanges 1 de ciervos (machos) con exostosis; $a=$ Cuadro $Q-1$, estrato III, fase IIIB; $b=$ Cuadro Q-3, estrato I, fase IIIB. Vista plantar.

Fig. 3. Falange 1 de un ciervo macho, rebajada y pulida. Cuadro $Q-2 B$, estrato III, fase IIIB; $a=$ vista plan tar; $b=$ vista axial.

Fig. 4. Falanges 1 de ovejas hembras rebajadas y pulidas; $a=$ Cuadro $Q-1$ estrato III, fase IIIB, vista axia1; $b=$ Corte $E$, fase IIIA, vista plantar.

Fig. 5. Falange 1 posterior de un caba11o; Cuadro $Q-2$ estrato II, fase IIIB(?); Vista dorsal.

Fig. 6. Metatarso de oveja conuna fractura soldada; Cuadro Q-4, estrato I, fase IIIB; Vista dorsal.

Fig. 7. Ombplatos de ciervos; $a=$ macho, cuadro Q-9, fase III; $\dot{b}=$ hembra corte $E$, fase IIIA. Ambos vista medial.

Fotos R. Zluwa figuras 2-7. 